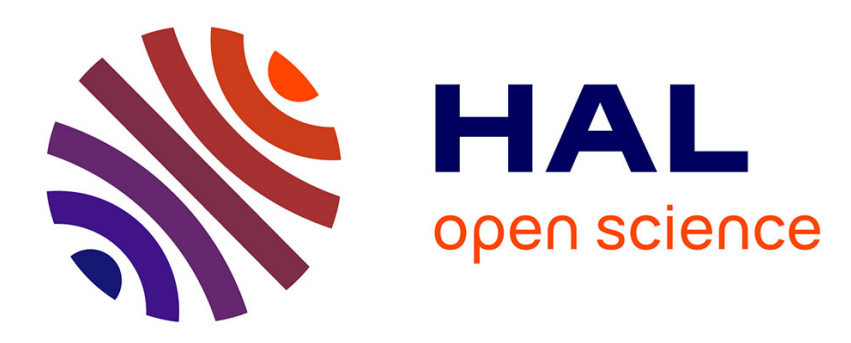

\title{
Dislocation Relaxation Processes due to Kink Migration
}

E. Hermida, A. Seeger, W. Ulfert

\section{- To cite this version:}

E. Hermida, A. Seeger, W. Ulfert. Dislocation Relaxation Processes due to Kink Migration. Journal de Physique IV Proceedings, 1996, 06 (C8), pp.C8-175-C8-178. 10.1051/jp4:1996835 . jpa-00254643

\section{HAL Id: jpa-00254643 https://hal.science/jpa-00254643}

Submitted on 1 Jan 1996

HAL is a multi-disciplinary open access archive for the deposit and dissemination of scientific research documents, whether they are published or not. The documents may come from teaching and research institutions in France or abroad, or from public or private research centers.
L'archive ouverte pluridisciplinaire HAL, est destinée au dépôt et à la diffusion de documents scientifiques de niveau recherche, publiés ou non, émanant des établissements d'enseignement et de recherche français ou étrangers, des laboratoires publics ou privés. 


\title{
Dislocation Relaxation Processes due to Kink Migration
}

\author{
E.B. Hermida(1), A. Seeger and W. Ulfert \\ Max-Planck Institut für Metallforschung, Institut für Physik and Universität Stuttgart, Institut für \\ Theoretische und Angewandte Physik, 70569 Stuttgart, Germany
}

\begin{abstract}
The interpretation of the Bordoni relaxation and of related relaxation phenomena in terms of the thermally activated, stress-assisted formation of kink pairs on dislocation lines is well established on assuming that the migration of kinks along dislocation lines is described by a high kink mobility $\mu_{k}$. This assumption, however, is not valid if the activation enthalpy for kink migration, $H_{m}$, is comparable with or even larger than the formation enthalpy of kink pairs or if even when small compared with the formation energy of kinks, $H_{m}$ is larger than the lowest thermal energies accessible in internal friction experiments. In those cases not only migration but also annihilation and trapping of thermal kink pairs may produce internal friction peaks.

The difference-differential equations governing the thermal kink pairs evolution along dislocation lines under the action of a homogeneous applied shear stress are set up and their timedependent solutions characterized by a set of relaxation times. It is shown how to obtain, from these solutions, the internal friction spectrum under conditions that are experimentally realized. Quantitative descriptions of geometrical kink migration and kink pair evolution are compared with selected experimental results.
\end{abstract}

\section{INTRODUCTION}

Since the first internal friction data measured in faced-centered-cubic (fcc) metals at low temperatures by Bordoni $[1,2]$ as well as the relaxation peaks observed by Niblett and Wilks [3,4], quite a profused work has been developed in order to explain this mechanical behaviour through the movement of dislocations as detailed in [5]. Not only damping peaks in fcc metals but also the $\alpha$ and $\gamma$ peaks in body-centered-cubic (bcc) metals - sometimes called refractory metals- have been explained by the kink pair formation (KPF) and geometrical kink migration (GKM) along screw and nonscrew dislocations [6]. Detailed theories [7-9] describe the relaxation of a dislocation with thermal and geometrical kinks by a set of relaxation times, assuming that the migration of kinks along the dislocation lines take place on a time scale that is either negligibly short or adequately described by a kink mobility $\mu_{k}$. Because of the high kink mobility they do not take into account how trapping of thermal kink pairs at the pinning points of the dislocation and annihilation of kinks of opposite signs affect the relaxation process.

In Section 2 kink pair formation and trapping and annihilation of thermal kinks with low mobility will be considered to determine the transient evolution of a dislocation segment while the relaxation parameters for the stationary state will be summarized in Section 3. Selected examples of IF measurements in materials with low kink mobility will be discussed in the last section.

(1) Alexander von Humboldt-Scholar 1994/1995. Permanent address: Unidad Act. Materiales, Comisión Nacional de Energía Atómica, Av. del Libertador 8250, 1429 Buenos Aires and Dto. de Física, Fac. de Ciencias Exactas y Naturales (UBA), Pabellón I, Ciudad Universitaria, 1428 Buenos Aires, Argentina 


\section{KINETICS OF THE KINK PAIR EVOLUTION}

It is considered a dislocation segment which extremes are at two pinning points separated by a distance $L$ in the same Peierls valley, that is to say, there are no geometrical kinks.

Since the dislocation is part of a dislocation network, its long-range interactions produce an internal stress $\sigma_{i}[10]$ in such a way that if at the time $t=0$ a constant resolved shear stress $\sigma$ is applied, the total stress results $\sigma_{t}=\sigma_{i}+\sigma$.

The temporal evolution of the probability of having $r$ kink pairs is described on taking into account on one side the kink pair formation rate per unit length [11]

$$
\Gamma=\pi \frac{4 D_{k}}{w_{k}^{2}} \frac{H_{k} \sigma a b}{(k T)^{2}} \mathrm{e}^{-2 H_{k} / k T}
$$

where $D_{k}=D_{o} \mathrm{e}^{-H_{m} / k T}$ is the diffusion coefficient, $T$ the absolute temperature, $k$ Boltzmann constant, $w_{k}$ the width of a single kink, $H_{k}$ the activation enthalpy for the formation of a single kink, $a$ the distance between Peierls valleys and $b$ Burgers vector, On the other hand also the annihilation and trapping rates to reduce the number of kinks pairs from $r$ to $r-1$,

$$
\tau_{r, r-1}^{-1}=\frac{2 D_{k}}{L^{*}} \frac{\sigma a b}{k T} r(r+1)
$$

are considered. $L^{*}$ denotes the effective length $L^{*}=(2 k T) /(\sigma a b) \sinh [(\sigma a b L) /(2 k T)]$ which can be understood as the lengthening of the dislocation segment when bowed out by the applied stress $\sigma$. Then, the temporal evolution of the probability of having $r$ kink pairs, $p_{r}$, can be described by the following system of difference-differential equations

$$
\partial_{\tilde{t}} p_{r}=q p_{r-1}-q p_{r}-r(r+1) p_{r}+(r+1)(r+2) p_{r+1} \quad, 0 \leq r \leq r_{\max }
$$

where $\partial_{\bar{t}}$ indicates the partial derivative with respect to $\tilde{t}=t f \tilde{\sigma}$, with $f=4 D_{k} / L^{* 2}$ and $\tilde{\sigma}=$ $\sigma_{t} a b L^{*} / 2 k T$. The probabilities $\left\{p_{r}\right\}$ verify the boundary condition $p_{-1}=0$ and the normalization condition $\sum_{r=0}^{r_{\max }} p_{r}=1$. The parameter of this system of difference-differential equations is the ratio between the creation rate of a kink pair and its "decay" (through annihilation or trapping)

$$
q=\pi\left(\frac{L^{*}}{w_{k}}\right)^{2} \frac{2 H_{k}}{k T} \mathrm{e}^{-\left(2 H_{k} / k T\right)}=\frac{2 \Gamma L^{*}}{\tau_{1,0}^{-1}}
$$

When $2 H_{k} / k T \gg 1, q$ tends to zero, i. e., there is practically no kink pair formation and the existing kinks can only migrate until they are annihilated by kinks of opposite sign or trapped at the pinning points of the dislocation segment. On the other hand, if $q \gg 1$, annihilation and trapping are negligible and the main relaxation process is due to the formation of kink pairs. The relaxation of a dislocation segment with kinks of low mobility in the interval of temperatures where $q \approx 1$ leads to a set of characteristic relaxation times which determination is detailed elsewhere [12].

\section{RELAXATION MECHANISMS IN THE STATIONARY STATE}

Once the KPE has reached its stationary state, the dislocation will move by the migration of $n_{g}$ geometrical kinks and the stationary evolution of $2\langle m\rangle$ thermal kinks. On assuming that the geometrical kinks present in a dislocation segment do not affect the KPE, i.e., there are no kink interactions, the area swept by the dislocation movement when an external stress $\sigma$ is applied can be evaluated as the area swept by geometrical kink migration (GKM) along the dislocation segment plus the area swept during the KPE. Therefore, the anelastic behaviour results the addition of the response due to both processes: GKM and KPE. 
On considering the position of the dislocation segment

$$
y(\sigma, x)=a \int_{0}^{x}\left[\rho_{-}(\sigma, u)-\rho_{+}(\sigma, u)\right] \mathrm{d} u .
$$

where $\rho_{ \pm}(\sigma, x)=n_{ \pm} / L^{*} \mathrm{e}^{-V_{ \pm}(x) / k T}$ is the equilibrium density of $n_{ \pm}$(positive or negative) kinks, the area swept by the dislocation between its initial and final position leads to the relaxation strength and the mean value of the ratio between the displacement of the dislocation and its velocity determines the relaxation time. Thus, it can be shown that GKM and KPE are characterized by the following relaxation times, $\tau$, and relaxation strengths, $\Delta,[12]$

$$
\begin{aligned}
\Delta_{G K M} & =\frac{\Lambda a^{2} b^{2} G L^{*}}{8 k T} n_{g} \\
\tau_{G K M} & =\frac{L^{2}}{8 D_{o}} \exp \left[H_{m} / k T\right] \\
\tau_{K P E} & =\frac{\sigma_{t} b L}{2 a S_{d} \Gamma}\left[1+\frac{\sqrt{q}}{4}\right] \\
\Delta_{K P E} & =\frac{\Lambda G b^{2} L^{2}}{12 S_{d}}
\end{aligned}
$$

on assuming that $\sigma \ll \sigma_{i}$. Since $D_{o}=\nu_{d} b^{2}$, where $\nu_{d}$ denotes the Debye frequency, it is expected that the pre-exponential factor of the relaxation time associated to the GKM is several orders of magnitude larger than the inverse of the Debye frequency. Similar results were found by Wüthrich [9].

\section{DISCUSSION}

The value of $H_{m}$ is comparable or even larger than the thermal energy in semiconductors such as $\mathrm{Ge}$ or Si. Jendrich and Haasen have measured the internal friction (IF) on deformed Ge at frequencies from 0.2 to $20 \mathrm{~Hz}$ and at temperatures between 150 and $820 \mathrm{~K}$ and found two maxima $\alpha$ and $\beta$. From the shifts of the peaks at different frequencies they determined that the relaxation frequencies of the $\alpha$ and $\beta$ processes follow an Arrhenius law with preexponential factors $\nu_{o \alpha}=(0.14$ to 15$) \times 10^{9} \mathrm{~s}^{-1}$ and $\nu_{o \beta} \approx 10^{13} \mathrm{~s}^{-1}$. Thus the first process can be associated to GKM and the second to KPE. Furthermore $H_{m}=H_{\alpha}=(1.108 \pm 0.01) \mathrm{eV}$ and $H_{\beta}=(2.07 \pm 0.2)=H_{m}+2 H_{k}-2 k T$ eV leading to $H_{k}=(.566 \pm .025) \mathrm{eV}$. Furthermore the relaxation strength of the GKM peak is approximately $10^{-2}$, leading to a mean dislocation length of $10^{-7} \mathrm{~m}$ and beween 5 and 10 geometrical kinks per dislocation segment. It is noticed that at the measured interval of temperatures it is $q<1$ and the distribution function of relaxation times due to the transient KPE reduces to a single process [11] which IF might be appreciated if $\omega \approx 10^{4} \mathrm{~s}^{-1}$, that is at higher frequencies than those used in [13].

Dynamic relaxation in $\mathrm{Nb}$ at very low temperatures $(<10 \mathrm{~K})$ is a second example of relaxation processes due to the migration of geometrical kinks with low mobility since both thermal energy and the enthalpy for migration of kinks are comparable. The IF peaks in $\mathrm{Nb}$ between 1.5 and $10 \mathrm{~K}$ at 80 and $240 \mathrm{kHz}$ were first observed and related to dislocation motions in 1967 by Kramer and Bauer in the superconducting, mixed and normal states of this material [14]. Another connection with GKM was proposed by Duffy and Umstattd who considered the migration of residual geometrical kinks to be the mechanism responsible for the low temperature background in $\mathrm{Nb}$ at $1 \mathrm{kHz}$ [15]. More recently Pal'-Val' et al. [16] reviewed the dynamical behaviour on acoustic relaxation in single crystal and polycrystalline samples of normal and superconducting $\mathrm{Nb}$ between 2 and $12 \mathrm{~K}\left(\omega \sim 10^{5}-10^{8} \mathrm{~s}^{-1}\right.$ ) in order to determine the influence of the sample structural characteristics on the parameters of the IF peak at low temperatures. From their data and previous experimental work Pal'-Val' et al. proposed an Arrhenius type law between the frequency of the IF and the temperature of its maximum and associated this process to the migration of screw dislocation kinks in the $<111>\{011\}$ 
slip system. They determined the following activation enthalpies for migration and pre-exponential factors: $H_{m n}=1.9 \mathrm{meV}, \nu_{o n}=2.1 .10^{10} \mathrm{~s}^{-1}, H_{m s}=2.7 \mathrm{meV}$ and $\nu_{o s}=1.1 .10^{10} \mathrm{~s}^{-1}$ ( $n$ and $s$ denote normal and superconducting states, respectively) and attributed the manifested differences to changes in the lattice parameters due to the Cooper condensation of electrons and to modifications of the electron shielding of the ion core in dislocation nuclei. However, below the critical temperature $T_{c}$ modifications in the elastic constants and changes in the lattice parameters of the material between the normal and superconducting states are of the order of $10^{-6}$ and $10^{-8}$, respectively. Hence, their contributions to changes in the relaxation times are expected to be negligible and our assumptions are more oriented to make an analogy between the kink movements and low-temperature quantum diffussion of light particles. This mechanism has been already mentioned by Takeuchi et al., who discussed a discrepancy of the Arrhenius rate equation in bcc metals at low temperatures in terms of the quantum tunnelling of a dislocation through a potential barrier [17]. Theoretical equations which describe tunnelling of light particles [18], tends in the limit of high temperatures to the classical transition-state-theory; particularly, the relaxation frequency follows an Arrhenius law at high temperatures. At extremely low temperatures, however, the tunnelling of light particles is characterized by a relaxation frequency which is independent of temperature and increases as the damping involved in the evolution of the system increases, for instance, as the sample goes from the superconducting to the normal state. This dependence of the relaxation frequency on temperature cannot be compared accurately with the actual data and new IF measurements both in the normal and superconducting states of $\mathrm{Nb}$ at other frequencies and even at lower temperatures would help to clarify the quatitative analysis of geometrical kinks tunelling.

\section{Acknowledgements}

The authors gratefully thank Dr. L. Schimmele for helpful discussions. One of the authors (E.H.) thanks the Alexander-von-Humboldt Foundation for a scholarship to work at the Max-Planck-Institut für Metallforschung, Institut für Physik and the members of this Institut for the kind hospitality.

\section{References}

[1] P. G. Bordoni, Ric. Sci. 19 (1949) 851-862.

[2] P. G. Bordoni, J. Acoust. Soc. Am. 26 (1954) 495-502.

[3] D. H. Niblett and J. Wilks, Phil. Mag. 1 (1956) 415-427.

[4] D. H. Niblett and J. Wilks, Phil. Mag. 2 (1957) 1427-1449.

[5] D. H. Niblett, in Physical Acoustics (Vol III A, W. P. Manson ed., Academic Press, New York (1966)). Ch.3.

[6] A. Seeger and C. Wüthrich, Il Nuovo Cimento 33 B (1976) 38-75.

[7] H. Engelke, phys. stat. sol. 36 (1969) 231-244.

[8] H. Engelke, phys. stat. sol. 36 (1969) 245-259.

[9] C. Wüthrich, Scripta Met. 9 (1975) 641-646.

[10] F. R. Nabarro, Z. S. Basinsky and D. B. Holt, Adv. in Phys. 13 (1964) 193-323.

[11] E. Mann, phys. stat. sol. (b) 111 (1982) 541-553.

[12] Élida B. Hermida and A. Seeger, to be published.

[13] U. Jendrich and P. Haasen, phys. stat. sol. (a) 108 (1988) 553-567.

[14] E. J. Kramer and C. L. Bauer, Phys. Rev. 163 (1967) 407-419.

[15] W. Duffy Jr. and R. Umstattd, J. Appl. Phys. 75 (1994) 4489-4495.

[16] P. P. Pal'-Val', D. V. Natsik and L. N. Pal'-Val', Low Temp. Phys. 21 (1995) 505-517.

[17] S. Takeuchi, T. Hashimoto and K. Maeda, Trans. JIM 23 (1982) 60-69.

[18] T. Regelmann, Master Thesis, Universität Stuttgart (1991). 